\title{
Exact Results for the Thermal and Magnetic Properties of Strong Coupling Ladder Compounds
}

\author{
Murray T. Batchelor, ${ }^{1}$ Xi-Wen Guan, ${ }^{1}$ Norman Oelkers, ${ }^{1}$ Kazumitsu Sakai, ${ }^{2}$ Zengo Tsuboi, ${ }^{2}$ and Angela Foerster ${ }^{3}$ \\ ${ }^{1}$ Department of Theoretical Physics, RSPhysSE and Centre for Mathematics and its Applications, MSI, \\ Australian National University, Canberra ACT 0200, Australia \\ ${ }^{2}$ Institute for Solid State Physics, University of Tokyo, Kashiwanoha 5-1-5, Kashiwa, Chiba, 277-8581, Japan \\ ${ }^{3}$ Instituto de Física da UFRGS, Avenue Bento Gonçalves, 9500, Porto Alegre, 91501-970, Brasil
}

(Received 11 September 2003; published 19 November 2003)

\begin{abstract}
We investigate the thermal and magnetic properties of the integrable $s u(4)$ ladder model by means of the quantum transfer matrix method. The magnetic susceptibility, specific heat, magnetic entropy, and high field magnetization are evaluated from the free energy derived via the recently proposed method of high temperature expansion for exactly solved models. We show that the integrable model can be used to describe the physics of the strong coupling ladder compounds. Excellent agreement is seen between the theoretical results and the experimental data for the known ladder compounds (5IAP) ${ }_{2} \mathrm{CuBr}_{4} \cdot 2 \mathrm{H}_{2} \mathrm{O}$, $\mathrm{Cu}_{2}\left(\mathrm{C}_{5} \mathrm{H}_{12} \mathrm{~N}_{2}\right)_{2} \mathrm{Cl}_{4}$, etc.
\end{abstract}

DOI: 10.1103/PhysRevLett.91.217202

PACS numbers: 75.10.Jm, 64.60.Cn, 75.40.Cx

The experimental realization of compounds with a ladderlike structure [1] has contributed to the intense interest in low-dimensional quantum systems. The existence of a spin gap, magnetization plateaux, quantum critical points, and superconductivity under hole doping are examples of key physical properties observed in the ladder compounds. Of particular importance are the properties under a magnetic field $H$. According to the perturbation theory result [1,2], the first-order terms for the zero temperature energy gap $\Delta$ and the critical field $H_{c 2}$ are given in terms of the rung $\left(J_{\perp}\right)$ and leg $\left(J_{\|}\right)$exchange couplings by $\Delta=J_{\perp}-J_{\|}$and $\mu_{B} g H_{c 2}=J_{\perp}+2 J_{\|}$. These results are in good agreement with the experimental data for strong coupling ladder compounds. However, the calculation of properties such as the full temperature phase diagram, the high field magnetization curve, and the specific heat provide a significant challenge.

We demonstrate here that the integrable $s u(4)$ ladder model $[3,4]$ is capable of describing the physics of the ladder compounds. Indeed, the thermodynamic Bethe ansatz (TBA) applied to the integrable $s u(4)$ ladder model predicts the critical fields $H_{c 1}=J_{\perp}-4 J_{\|} / \gamma$ and $\mu_{B} g H_{c 2}=J_{\perp}+4 J_{\|} / \gamma$, where $\gamma$ is a rescaling parameter, which are also good fits for the strong coupling compounds [5]. Very recently the high temperature expansion (HTE) method [6,7] suggested

a way to calcuate the full thermodynamic properties of integrable models from the so-called $T$ system [8] appearing in the quantum transfer matrix (QTM) formalism $[9,10]$. Here we extend this approach to the integrable ladder model to derive the thermal and magnetic properties of the strong coupling ladder compounds. We compare our results with the experimental data obtained for the compounds $\mathrm{Cu}_{2}\left(\mathrm{C}_{5} \mathrm{H}_{12} \mathrm{~N}_{2}\right)_{2} \mathrm{Cl}_{4}[11,12]$ and (5IAP) ${ }_{2} \mathrm{CuBr}_{4} \cdot 2 \mathrm{H}_{2} \mathrm{O}$ [13].

The Hamiltonian of the ladder model is $[3-5,14]$

$$
\begin{aligned}
\mathcal{H} & =\frac{J_{\|}}{\gamma} \mathcal{H}_{\mathrm{leg}}+J_{\perp} \sum_{j=1}^{L} \vec{S}_{j} \vec{T}_{j}-\mu_{B} g H \sum_{j=1}^{L}\left(S_{j}^{z}+T_{j}^{z}\right), \\
\mathcal{H}_{\mathrm{leg}} & =\sum_{j=1}^{L}\left(\vec{S}_{j} \vec{S}_{j+1}+\vec{T}_{j} \vec{T}_{j+1}+4 \vec{S}_{j} \vec{S}_{j+1} \vec{T}_{j} \vec{T}_{j+1}\right),
\end{aligned}
$$

where $\vec{S}_{j}$ and $\vec{T}_{j}$ are Heisenberg operators, $\mu_{B}$ is the Bohr magneton, and $g$ is the Landé factor. Throughout, $L$ is the number of rungs and periodic boundary conditions are imposed. In the strong coupling limit, the contribution to the low-temperature physics from the multibody term in $\mathcal{H}_{\text {leg }}$ is minimal and, as a consequence, the integrable ladder Hamiltonian exhibits similar critical behavior to the standard Heisenberg ladder [5]. We adapt the model into the QTM method [9]. The eigenvalue of the QTM (up to a constant) is obtained by the nested Bethe ansatz to be

$$
\begin{aligned}
T_{1}^{(1)}\left(v, v_{i}^{(a)}\right)= & e^{\beta \mu_{1}} \phi_{-}(v-i) \phi_{+}(v) \frac{Q_{1}\left(v+\frac{1}{2} i\right)}{Q_{1}\left(v-\frac{1}{2} i\right)}+e^{\beta \mu_{2}} \phi_{-}(v) \phi_{+}(v) \frac{Q_{1}\left(v-\frac{3}{2} i\right) Q_{2}(v)}{Q_{1}\left(v-\frac{1}{2} i\right) Q_{2}(v-i)} \\
& +e^{\beta \mu_{3}} \phi_{-}(v) \phi_{+}(v) \frac{Q_{2}(v-2 i) Q_{3}\left(v-\frac{1}{2} i\right)}{Q_{2}(v-i) Q_{3}\left(v-\frac{3}{2} i\right)}+e^{\beta \mu_{4}} \phi_{-}(v) \phi_{+}(v+i) \frac{Q_{3}\left(v-\frac{5}{2} i\right)}{Q_{3}\left(v-\frac{3}{2} i\right)} .
\end{aligned}
$$

In this equation the chemical potential terms are $\mu_{1}=J_{\perp} / 2, \mu_{2}=\mu_{B} g H, \mu_{3}=0$, and $\mu_{4}=-\mu_{B} g H$, with $\phi_{ \pm}(v)=$ $\left(v \pm i u_{N}\right)^{N / 2}$. The inhomogeneity parameter $u_{N}=-\left(J_{\|} \beta / \gamma N\right)$, with $Q_{a}[v)=\prod_{i=1}^{M^{(a)}}\left(v-v_{i}^{(a)}\right]$, for $a=1,2$, 3. Here $N$ denotes the Trotter-Suzuki number. The fused $T_{m}^{(a)}$ system [8], which denotes the row-to-row transfer matrix with fusion type $(a, m)$ in the auxiliary space carrying the $m$-fold symmetric tensor of the $a$ th fundamental representation of the 
$s u(4)$ algebra, is essentially generated by the QTM eigenvalue $T_{1}^{(1)}$ in (2). Thus $T_{1}^{(1)}$ can be embedded into the fused $T_{m}^{(a)}$ system. The analytic nonzero and constant asymptotic properties of the normalized $\tilde{T}_{m}^{(a)}(v)$ system suggest the expansion ansatz

$$
\lim _{N \rightarrow \infty} \tilde{T}_{1}^{(a)}(v)=\exp \left[\sum_{n=0}^{\infty} b_{n}^{(a)}(v)\left(\frac{J_{\|}}{\gamma T}\right)^{n}\right]
$$

with $b_{n}^{(a)}(v)=\sum_{j=0}^{n-1} c_{n, j}^{(a)} v^{2 j} /\left[v^{2}+(a+1)^{2} / 4\right]^{n}$. The QTM eigenvalue satisfies a set of the nonlinear integral equations [7]

$$
\begin{aligned}
\tilde{T}_{1}^{(a)}(v)= & Q_{1}^{(a)}+\oint_{C_{m}^{(a)}} \frac{d y}{2 \pi i} \frac{1}{v-y-\beta_{1}^{(a)}}\left[\frac{\tilde{T}_{1}^{(a-1)}\left(y+\beta_{1}^{(a)}-\frac{1}{2} i\right) \tilde{T}_{1}^{(a+1)}\left(y+\beta_{1}^{(a)}-i\right)}{\tilde{T}_{1}^{(a)}\left(y+\beta_{1}^{(a)}-i\right)}\right] \\
& +\oint_{\bar{C}_{m}^{(a)}} \frac{d y}{2 \pi i} \frac{1}{v-y+\beta_{1}^{(a)}}\left[\frac{\tilde{T}_{1}^{(a-1)}\left(y-\beta_{1}^{(a)}+\frac{1}{2} i\right) \tilde{T}_{1}^{(a+1)}\left(y-\beta_{1}^{(a)}+\frac{1}{2} i\right)}{\tilde{T}_{1}^{(a)}\left(y-\beta_{1}^{(a)}+i\right)}\right], \quad a=1,2,3 .
\end{aligned}
$$

Following Ref. [7], the coefficients $c_{n, j}^{(a)}$ can be obtained recursively from Eq. (4) with initial conditions $b_{0}^{(a)}=$ $\ln Q_{1}^{(a)}$, where $Q_{1}^{(a)}$ are constants related to the chemical potential terms via $\lim _{N \rightarrow \infty} \lim _{|v| \rightarrow \infty} \tilde{T}_{1}^{(a)}(v)=Q_{1}^{(a)}$ with $Q_{1}^{(0)}=1$ and $Q_{1}^{(4)}=\exp \left(J_{\perp} / 2 T\right)$. In this way the spin ladder free energy $f(T, H)=-T \ln T_{1}^{(1)}$ can be expanded in powers of $J_{\|} / \gamma T$. For the first few orders we have

$$
\begin{aligned}
-\frac{1}{T} f(T, H)= & \ln \left(2 B_{\epsilon, 1}\right)+A\left(\frac{J_{\|}}{\gamma T}\right) \\
& +\frac{3}{2}\left(A-A^{2}+\frac{1}{2} \frac{\epsilon B_{1, \epsilon}}{B_{\epsilon, 1}^{3}}\right)\left(\frac{J_{\|}}{\gamma T}\right)^{2},
\end{aligned}
$$

where $A=B_{\epsilon, 0}\left(1+2 B_{0,1}\right) / B_{\epsilon, 1}^{2}$ with $\epsilon=\exp \left(J_{\perp} / 2 T\right)$ and

$$
B_{x, y}=x \cosh \left(\frac{J_{\perp}}{2 T}\right)+y \cosh \left(\frac{\mu_{B} g H}{T}\right) .
$$

We find that the analytic expression (5) is sufficiently accurate to evaluate the model's thermodynamics. Nevertheless, we have considered the HTE up to fifth order.

The experimental measurements of the susceptibility and magnetization of the compound (5IAP) ${ }_{2} \mathrm{CuBr}_{4} \cdot 2 \mathrm{H}_{2} \mathrm{O}$ [13] (abbreviated B5i2aT) suggest a spin ladder with exchange couplings $J_{\perp}=13.0 \mathrm{~K}$ and $J_{\|}=1.15 \mathrm{~K}$. From the HTE for the integrable model we find that the values $J \perp=13.3$ Kand $\mathrm{J} \|=1.15 \backslash ; \mathrm{K}$ with rescaling parameter $\gamma=4$ give excellent fits to both the susceptibility and magnetization [15]. The temperature dependence of the susceptibility is shown in Fig. 1. The agreement with the theoretical curve derived from the HTE is clearly excellent. The typical rounded peak for low magnetic field, characteristic of a low-dimensional antiferromagnet, is observed around $8.1 \mathrm{~K}$, in excellent agreement with the experimentally estimated value of $8 \mathrm{~K}$. The inset in Fig. 1 shows the low-temperature behavior of the susceptibility, which is in excellent agreement with the experimental data.
The theoretical curves for the high field magnetization shown in Fig. 2 for different temperatures are also in good agreement with the experimental values. The field dependent magnetization curve predicts the lowtemperature phase diagram as well as the magnetization plateaus. For very low temperature the rung singlet forms a dimerized ground state if the magnetic field is below the critical field $H_{c 1}$. The length of the antiferromagnetic correlation is finite while the triplet state is gap full. For finite temperatures the triplet excitations are also involved in the gapped phase. This can be observed in the high field magnetization curves for $T=1.59 \mathrm{~K}$ and $T=$ $4.35 \mathrm{~K}$ in Fig. 2. At the critical field $H_{c 1}$, the gap is closed with $\mu_{B} g H_{c 1}=\Delta$. If the magnetic field is above the critical point $H_{c 1}$, the lower triplet component becomes

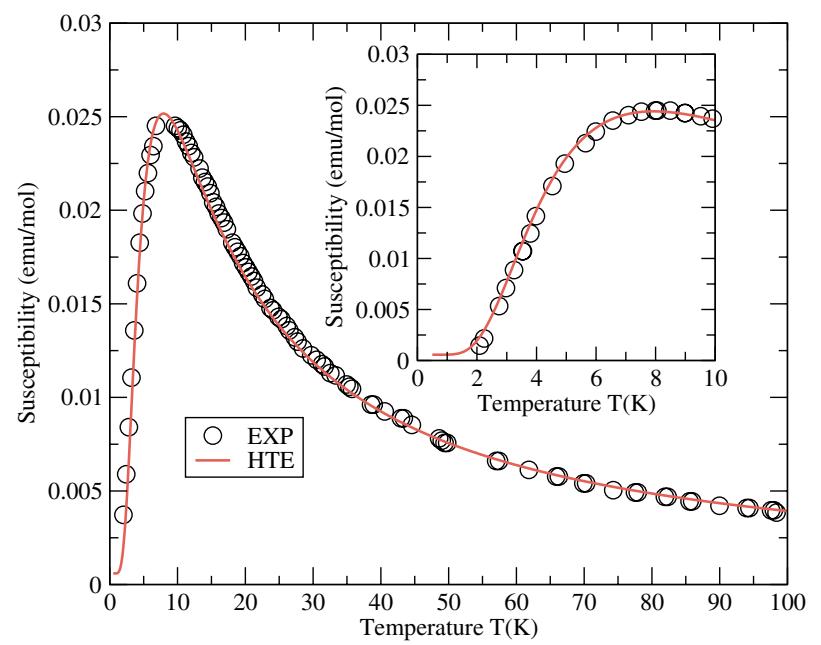

FIG. 1 (color online). The susceptibility versus temperature for B5i2aT at $H=1 \mathrm{~T}$ [13]. The solid line denotes the susceptibility evaluated directly from the HTE. A parameter fit suggests the coupling constants $J_{\perp}=13.3 \mathrm{~K}$ and $J_{\|}=1.15 \mathrm{~K}$ with $\gamma=4, g=2.1$, and $\mu_{B}=0.672 \mathrm{~K} / \mathrm{T}$. The inset shows the same fit to the susceptibility at low temperature. 


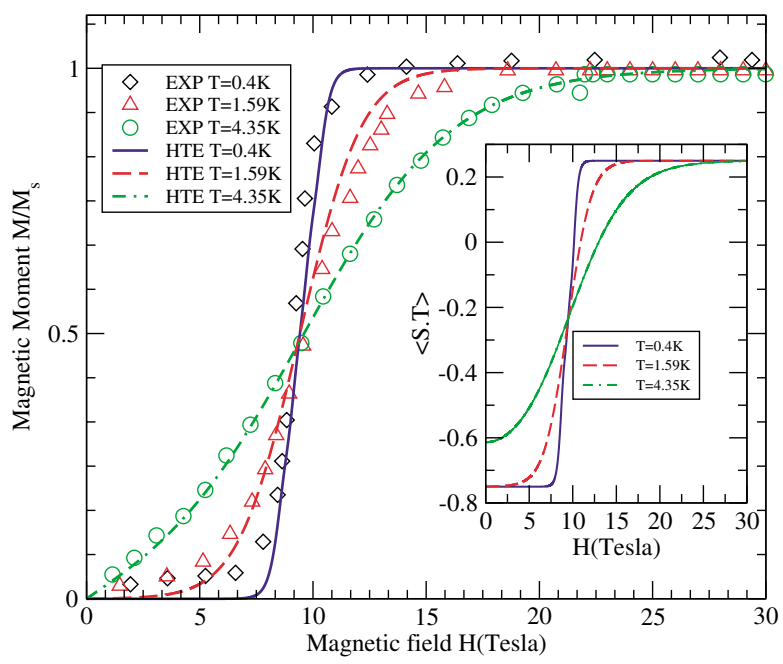

FIG. 2 (color online). Magnetization versus magnetic field for B5i2aT [13] with the same constants as in Fig. 1. The discrepancy in the magnetization curves at $T=0.4 \mathrm{~K}$ and $T=1.59 \mathrm{~K}$ is due to paramagnetic impurities which become negligible for higher temperature. The inset shows the one-point correlation function versus magnetic field.

involved in the ground state. For zero temperature, it can be rigorously shown that the other two higher triplet components do not become involved in the ground state [5]. It follows that the strong coupling ladder can be mapped to the $X X Z$ Heisenberg chain with an effective magnetic field term $[2,16]$. The magnetization increases almost linearly with the field towards the critical point $H_{c 2}$, where the ground state is fully polarized. At $T=$ $0.4 \mathrm{~K}$, the HTE magnetization curve indicates $H_{c 1} \approx$ $8.3 \mathrm{~T}$ and $H_{c 2} \approx 10.5 \mathrm{~T}$, which are in excellent agreement with the experimental estimates of 8.4 and $10.4 \mathrm{~T}$. The experimental magnetization in the singlet ground state at low temperature appears to be nonzero. This nonzero magnetization is attributed to paramagnetic impurities, which drive the low-temperature deviation between the experimental and the theoretical curves in Fig. 2.

The inflection point is clearly visible in the experimental magnetization curves [13]. This point is also evident in the theoretical curves at $\mu_{B} g H \approx J_{\perp}$ where the magnetization moment is $\frac{1}{2}$. The physical meaning of the inflection point is that the probabilities of the singlet and the triplet states $|\uparrow \uparrow\rangle$ in the ground state are equal. Therefore, for the strong coupling ladder compounds at zero temperature the one-point-correlation function $\left\langle S_{j} \cdot T_{j}\right\rangle=$ $-\frac{3}{4}$ lies in a gapped singlet ground state, which indicates an ordered dimer phase, while $\left\langle S_{j} \cdot T_{j}\right\rangle=\frac{1}{4}$ in the fully polarized ferromagnetic phase. However, in a Luttinger liquid phase, we find $\left\langle S_{j} \cdot T_{j}\right\rangle=-\frac{3}{4}+S^{z}$. At low temperatures $T \ll J_{\perp}$, the one-point correlation function is given by $\left\langle S_{j} \cdot T_{j}\right\rangle=\frac{1}{4}+\left[\left(d / d J_{\perp}\right) f(T, H)\right]_{T}$. The field-induced quantum phase transitions can be clearly seen from the one-point correlation function curve shown in the inset of Fig. 2.

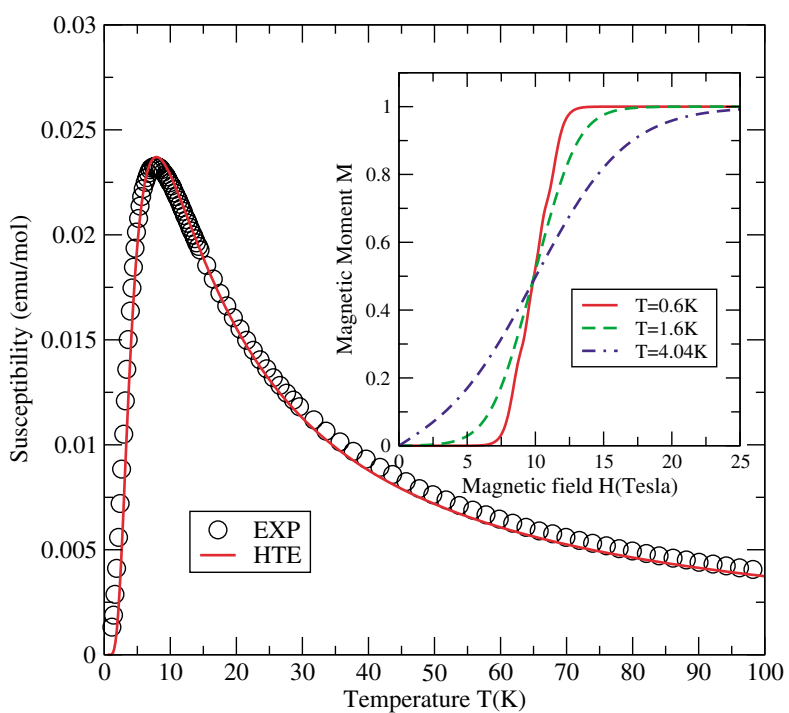

FIG. 3 (color online). Susceptibility versus temperature for the compound $\mathrm{Cu}(\mathrm{Hp}) \mathrm{Cl}$ [12]. The solid line denotes the susceptibility evaluated directly from the HTE with $\mu_{B}=$ $0.672 \mathrm{~K} / \mathrm{T}, J_{\perp}=13.5 \mathrm{~K}, J_{\|}=2.4 \mathrm{~K}, \gamma=5$, and $g=2.03$. The inset shows the magnetization versus magnetic field at different temperature. At $T=0.6 \mathrm{~K}$, the critical fields are $H_{c 1} \approx 7.8 \mathrm{~T}$ and $H_{c 2} \approx 13.0 \mathrm{~T}$, in good agreement with the experimental results $[11,12]$.

We turn now to the ladder compound $\mathrm{Cu}_{2}\left(\mathrm{C}_{5} \mathrm{H}_{12} \mathrm{~N}_{2}\right)_{2} \mathrm{Cl}_{4}$ [11,12] [abbreviated $\mathrm{Cu}(\mathrm{Hp}) \mathrm{Cl}$ ]. In Fig. 3, we show the zero field magnetic susceptibility curve obtained from the fifth order HTE free energy. A full fit with the experimental data suggests the coupling constants $J_{\perp}=13.5 \mathrm{~K}, J_{\|}=2.4 \mathrm{~K}$, and $\gamma=5$. The effect of the magnetic field is to lift the susceptibility in the low-temperature regime. We notice that there is a



FIG. 4 (color online). Specific heat versus temperature for the compound $\mathrm{Cu}(\mathrm{Hp}) \mathrm{Cl}$ [12] with the same constants as in Fig. 3. The inset shows the field dependent entropy versus temperature. 


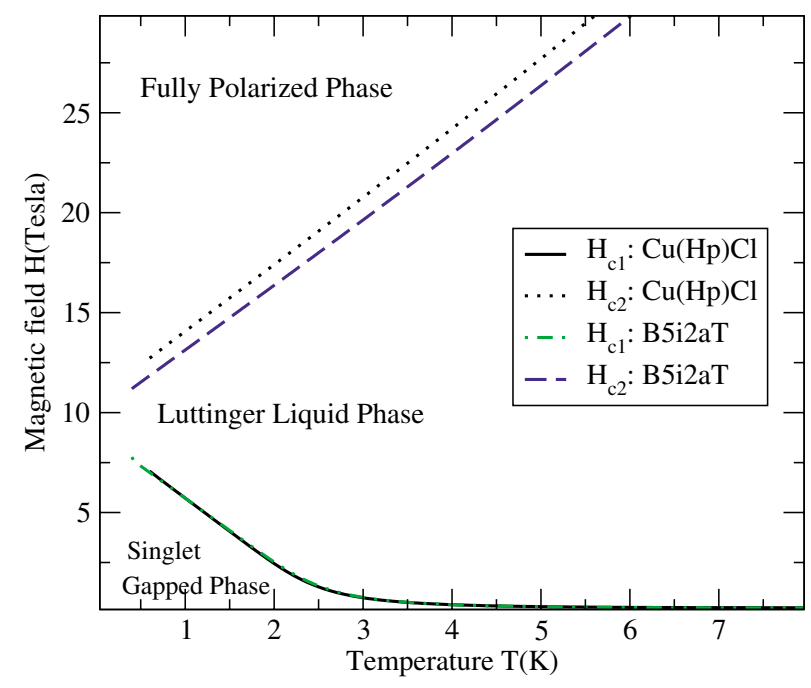

FIG. 5 (color online). Phase diagrams for the compounds B5i2aT and $\mathrm{Cu}(\mathrm{Hp}) \mathrm{Cl}$.

discrepancy with the zero temperature TBA result for $H_{c 2}$ [5] due to the presence of strong exchange coupling along the legs. For finite temperature this discrepancy is smaller. The inset curves in Fig. 3 show the high field magnetization for temperatures $T=0.6,1.6$, and $4.04 \mathrm{~K}$. We observe the fact that the critical points are $H_{c 1} \approx 7.8 \mathrm{~T}$ and $H_{c 2} \approx 13.0 \mathrm{~T}$, which are in good agreement with the experimental values [11,12]. It is also obvious that the finite temperature causes a spin flip in the gapped ground state.

The specific heat curves in Fig. 4 for $H=0 \mathrm{~T}$ and $H=$ $4 \mathrm{~T}$ indicate that the HTE result also agrees satisfactorily with the experimental data [12]. In the absence of a magnetic field, a rounded peak indicating short range ordering is observed around $4.5 \mathrm{~K}$. For temperatures $T<$ $4.5 \mathrm{~K}$, there is an exponential decay due to an ordered phase. The humps become smaller as the magnetic field increases. For the $H=4 \mathrm{~T}$ curve a peak is observed at around $4 \mathrm{~K}$. As to be expected, there appears to be a small deviation from the experimental data at very low temperatures. The inset of Fig. 4 shows that the entropy curves for magnetic fields $H=0 \mathrm{~T}$ and $H=4 \mathrm{~T}$ are also in agreement with the experimental data [12].

The full phase diagram for the two compounds is shown in Fig. 5. The slopes of the critical curves indicate that the estimated values of $H_{c 1}$ and $H_{c 2}$ at $T=0 \mathrm{~K}$ coincide with the TBA results [5]. We have also examined other strong coupling compounds. Comparison with the experimental data for the compound BIP-BNO [17] suggests the coupling constants $J_{\perp}=75 \mathrm{~K}, J_{\|}=$ $15 \mathrm{~K}$ with $\gamma=6.0$ and $g=2.0$. For the compound $\left[\mathrm{Cu}_{2}\left(\mathrm{C}_{2} \mathrm{O}_{4}\right)\left(\mathrm{C}_{10} \mathrm{H}_{8} \mathrm{~N}_{2}\right)_{2}\right]\left(\mathrm{NO}_{3}\right)_{2}$ [18] we find $J_{\perp}=515 \mathrm{~K}$, $J_{\|}=40 \mathrm{~K}$ with $\gamma=5.0$ and $g=2.14$. The respective spin excitation gaps, $\Delta \approx 52 \mathrm{~K}$ and $\Delta \approx 460 \mathrm{~K}$, are also in good agreement with the experimental values.
The application of the HTE method to other ladder models, such as the mixed-spin ladders, should now be straightforward. We also note that the HTE method predicts a fractional magnetization plateau with respect to different Landé $g$ factors in the $s u(4)$ spin-orbital model, which coincides with the Bethe ansatz [19] and TBA [20] results.

This work has been supported by the Australian Research Council. K. S. is supported in part by JSPS. N. O. thanks DAAD. A. F. thanks FAPERGS and CNPq. We thank H.-Q. Zhou and Z.-J. Ying for helpful discussions.

[1] For reviews, see E. Dagotto and T. M. Rice, Science 271, 618 (1996); E. Dagotto, Rep. Prog. Phys. 62, 1525 (1999).

[2] M. Reigrotzki, H. Tsunetsugu, and T. M. Rice, J. Phys. Condens. Matter 6, 9235 (1994).

[3] Y. Wang, Phys. Rev. B 60, 9236 (1999).

[4] M. T. Batchelor and M. Maslen, J. Phys. A 32, L377 (1999); M. T. Batchelor, J. de Gier, and M. Maslen, J. Stat. Phys. 102, 559 (2001).

[5] M. T. Batchelor, X.-W. Guan, A. Foerster, and H.-Q. Zhou, New J. Phys. 5, 107 (2003).

[6] M. Shiroishi and M. Takahashi, Phys. Rev. Lett. 89, 117201 (2002).

[7] Z. Tsuboi, J. Phys. A 36, 1493 (2003).

[8] A. Kuniba, T. Nakanishi, and J. Suzuki, Int. J. Mod. Phys. A 9, 5215 (1994).

[9] M. Suzuki, Phys. Rev. B 31, 2957 (1985); A. Klümper, Ann. Phys. (Leipzig) 1, 540 (1992); G. Jüttner, A. Klümper, and J. Suzuki, Nucl. Phys. B487, 650 (1997).

[10] A different QTM method has been applied numerically to the standard Heisenberg ladder in M. Troyer, H. Tsunetsugu, and D. Würtz, Phys. Rev. B 50, 13515 (1994).

[11] G. Chaboussant et al., Phys. Rev. B 55, 3046 (1997); Phys. Rev. Lett. 79, 925 (1997); 80, 2713 (1998).

[12] M. Hagiwara, H. A. Katori, U. Schollwöck, and H.-J. Mikeska, Phys. Rev. B 62, 1051 (2000).

[13] C. P. Landee, M. M. Turnbull, C. Galeriu, J. Giantsidis, and F. M. Woodward, Phys. Rev. B 63, 100402 (2001).

[14] The multibody term arises naturally in other contexts, see, e.g., A. A. Nersesyan and A. M. Tsvelik, Phys. Rev. Lett. 78, 3939 (1997); A. K. Kolezhuk and H.-J. Mikeska, Phys. Rev. Lett. 80, 2709 (1998).

[15] An overall unit constant in the susceptibility needs to be chosen to match the experimental scaling as the $T$ system is determined only up to a multiplicative factor.

[16] G. Chaboussant et al., Eur. Phys. J. B 6, 167 (1998).

[17] K. Katoh, Y. Hosokoshi, K. Inoue, and T. Goto, J. Phys. Soc. Jpn. 69, 1008 (2000).

[18] Z. Honda, Y. Nonomura, and K. Katsumata, J. Phys. Soc. Jpn. 66, 3689 (1997).

[19] S.-J. Gu, Y.-Q. Li, and H.-Q. Zhou, cond-mat/0308432.

[20] Z.-J. Ying, A. Foerster, X.-W. Guan, B. Chen, and I. Roditi, cond-mat/0308443. 